\title{
Fraunhofer Diffraction of Light by Human Enamel
}

W.J. O'BRIEN

Department of Biomaterials, School of Dentistry, University of Michigan, Ann Arbor, Michigan 48109-1078

Fraunhofer diffraction patterns of human enamel samples were photographed with a helium-neon laser beam $(\lambda=633 \mathrm{~nm})$. The firstorder diffraction angle was in reasonable agreement with a prediction based upon enamel prisms acting as a two-dimensional grating. These results support the hypothesis that enamel diffracts light because of the periodic structure of enamel prisms with interprismatic spaces, which act as slits.

J Dent Res 67(2):484-486, February, 1988

\section{Introduction.}

The optical properties of enamel have been an area of research interest with two main practical applications. First, the transmission and scattering of light are related to the detection of early demineralization and incipient caries (Spitzer and Ten Bosch, 1977). The appearance of white spots on demineralized enamel has been explained on the basis of increased lightscattering due to porosity. Also, the mechanism of light transmission through enamel has recently been studied to aid in our understanding of the color of teeth. The appearance of a tooth is the result of diffuse reflectance from the dentin through enamel acting as a light-scattering layer. The translucency of enamel has been found to be wavelength- and moisture-content-dependent (Brodbelt et al., 1981). Finally, the simulation of the appearance of teeth with porcelain crowns requires a better understanding of light-scattering by enamel ( $O^{\prime}$ Brien, 1985).

The periodic structure of enamel indicates that it will diffract light in addition to scattering it, as shown by Groenhuis et al. (1984). The prisms of human enamel in the model used here consist of cylindrical crystals which run parallel to each other from the dentin-enamel junction to the outer surface. The prisms have a diameter of about $5 \mu \mathrm{m}$ (Arends and Ten Cate, 1981) but are "keyhole"-shaped. Although the composition and structure of interprismatic material is still a matter of debate, for the purposes of this work it is assumed that the interface between the prisms is $0.1 \mu \mathrm{m}$ (Arends and Ten Cate, 1981). This is analogous to a diffraction grating when light is directed perpendicular to the enamel surface and roughly parallel to the prisms. The light will be diffracted as it passes through the interfacial spaces, which act as "slits".

The purpose of this study was to obtain the diffraction pattern of coherent light passing through human enamel and compare it with the predictions based upon a diffraction grating.

The prediction of Fraunhofer diffraction patterns can be obtained mathematically based upon a model of a diffraction grating. The intensity function of a Fraunhofer diffraction pattern for an ideal grating with multiple narrow slits is shown in Fig. 1 (Hecht, 1975). The principal maxima are given by:

$$
\sin \theta=m \lambda / d
$$

where $\theta$ is the angle from the central spot to the diffracted

Received for publication April 10, 1987

Accepted for publication October 21, 1987

This investigation was supported in part by USPHS Research Grants DEO2731 and DEO5423 from the National Institute of Dental Research, National Institutes of Health, Bethesda, MD 20892.

\section{INTENSITY FUNCTION}

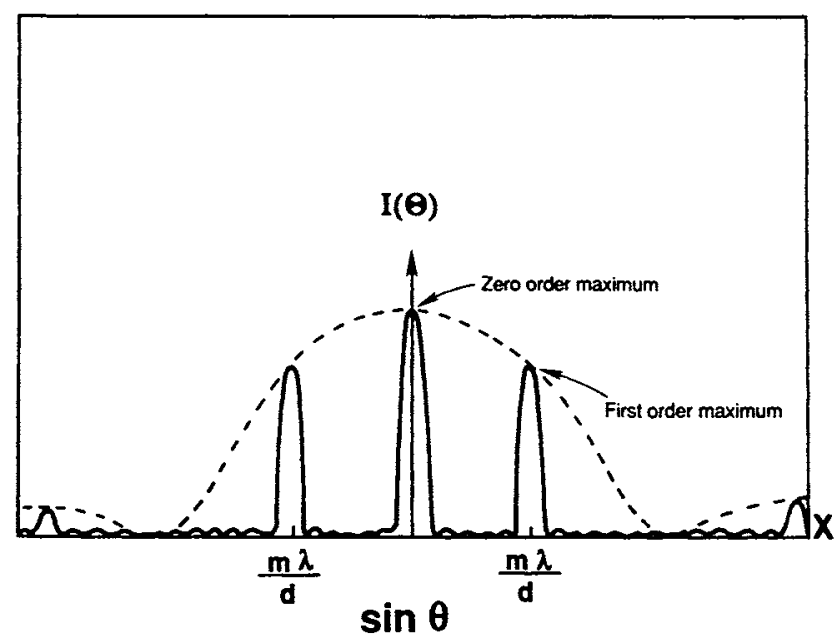

Fig. 1-Calculated intensity pattern for Fraunhofer diffraction of light by multiple slits (after Hecht, 1975).

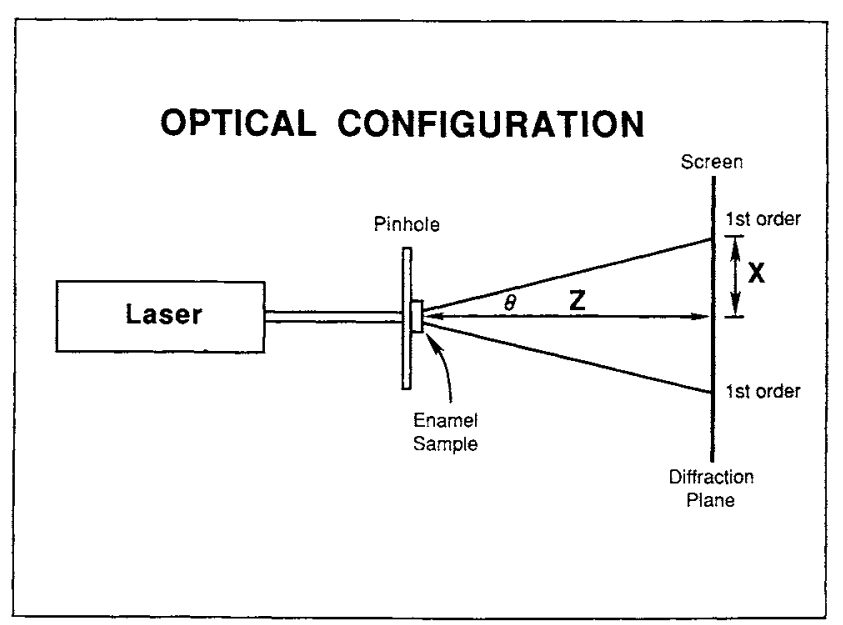

Fig. 2-Experimental arrangement for production of Fraunhofer diffraction.

maxima, $\mathrm{m}$ is the order of diffraction, $\mathrm{d}$ is the center-to-center prism distance, and $\lambda$ is the wavelength of light.

Using the following data of Arends and Ten Cate (1981) for the center-to-center prism distance of $5 \mu \mathrm{m}$ for $\mathrm{d}$ and a wavelength of $0.633 \mu \mathrm{m}$ corresponding to that of a helium neon laser (red) light, one can calculate the location of the firstorder peak from equation (1) to be $\pm 7^{\circ} 20^{\prime}$.

\section{Materials and methods.}

Diffraction pattern.-Fraunhofer, or far-field diffraction, patterns were obtained by the arrangement shown in Fig. 2 . Planoparallel enamel slices approximately $0.5 \mathrm{~mm}$ were cut and polished from the facial surfaces of extracted human incisors. A helium-neon laser was aligned with a $50-\mu \mathrm{m}$-diam- 

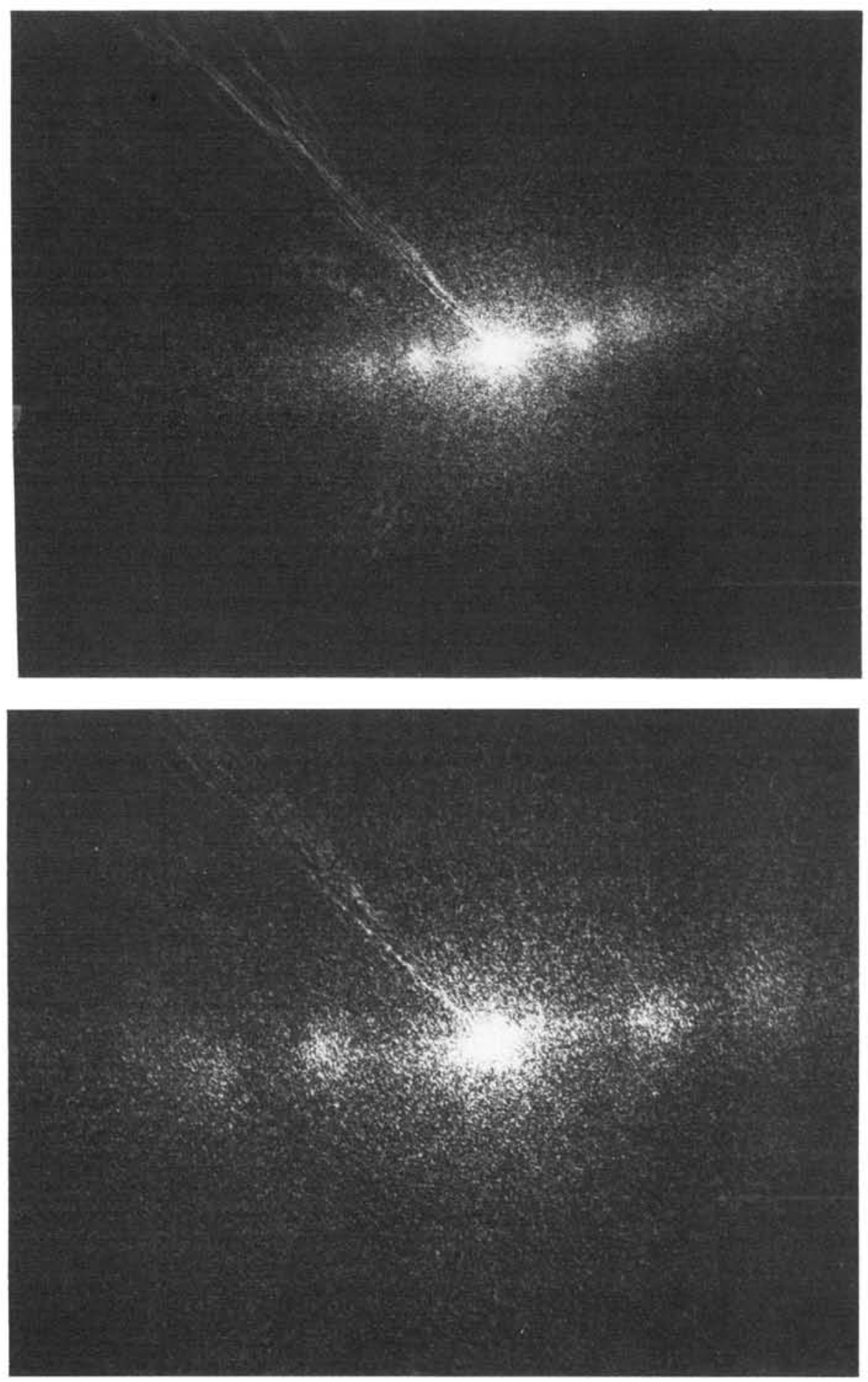

Fig. 3-Fraunhofer diffraction pattern of human dental enamel at distance $(\mathrm{z})$ of 100 $\mathrm{mm}$ from sample. eter pinhole. Samples wet with distilled water were mounted in contact with the pinhole. (Strong diffraction patterns could not be obtained with dry samples.) Diffraction patterns were photographed at distances of $20,40,100$, and $200 \mathrm{~mm}$ away from the enamel samples. The angle $\theta$ indicates the position of the first-order diffraction spot.

\section{Results.}

The Fraunhofer diffraction patterns at screen distances of 100 and $200 \mathrm{~mm}$ were the clearest and are shown in Figs. 3 and 4 . Here the first- and second-order diffraction maxima are clearly seen. Measurements of the angle are from the center. The pattern is similar to one produced by the scattering of diffraction with a grating of multiple slits. The angular dependence of scattering first observed by Groenhuis et al. (1984) is supported. The position of the first-order diffraction spots was measured, and the diffraction angle, $\theta$, was calculated from

$$
\tan \theta=\mathrm{x} / \mathrm{z}
$$

where $\mathrm{x}$ is the distance from the central spot to the first-order 
diffraction spot, and $z$ is the specimen-screen distance. The average measured angle was $6^{\circ} 48^{\prime}$. This compares with an angle of $7^{\circ} 20^{\prime}$ calculated from equation 1 based upon a prismto-prism distance of $5 \mu \mathrm{m}$. This may be considered reasonable agreement considering the assumptions made in the calculations. First of all, enamel structure is a three-dimensional distribution of enamel prisms rather than a two-dimensional grating pattern. Also, it is possible that the prism center-to-center distance is greater than $5 \mu \mathrm{m}$, as assumed in the calculation. The measured angle of $6^{\circ} 48^{\prime}$ indicates a prism distance of $5.7 \mu \mathrm{m}$.

\section{Discussion.}

The demonstration that enamel diffracts light has two main applications. First of all, Fraunhofer diffraction offers another research method for study of the structure of enamel. Diffraction of light is sensitive to the center-to-center prism distance. Another application is to aid in understanding of the appearance properties of dental enamel. Since diffraction produces strong scattering of light, it has an effect on the transmission properties. The wavelength- and moisture-dependence of light transmission by enamel, shown by Brodbelt et al. (1981), may be partly explained by diffraction. This is implied, since diffraction decreases with increasing wavelength (equation 1), and strong patterns could only be obtained with wet samples.
The model presented in this paper could be further refined to correspond more directly with the irregular structure of enamel.

\section{Acknowledgments.}

The advice and assistance of Kenneth M. Boenke, William M. Johnston, and Russell M. Pon on this project are gratefully acknowledged.

\section{REFERENCES}

ARENDS, J. and TEN CATE, J.M. (1981): Tooth Enamel Remineralization, $J$ Cryst Growth 53:135-147.

BRODBELT, R.H.W.; O'BRIEN, W.J.; FAN, P.L.; FRAZIER, J.G.; and YU, R. (1981): Translucency of Human Enamel, J Dent Res 60:1749-1753.

GROENHUIS, R.A.J.; TEN BOSCH, J.J.; and FERWERDA, H.A. (1984): Optical Properties of Dental Enamel, J Dent Res 63:168, Abst. No. S21 (AADR).

HECHT, E. (1975): Theory and Problems of Optics, New York: McGraw-Hill.

O'BRIEN, W.J. (1985): Double Layer Effect and Other Optical Phenomena Related to Esthetics, Dent Clin N Am 29: 667-672.

SPITZER, D. and TEN BOSCH, J.J. (1977): Luminescence Quantum Yields of Sound and Carious Dental Enamel, Calcif Tissue Res 24:249-251. 